\title{
Limited Central Hepatectomy for Centrally Located Tumors: Is There a Place for Standardization?
}

\author{
Florin Botea ${ }^{1}$, Alexandru Barcu ${ }^{1}$, Adina Croitoru², Dana Tomescu ${ }^{3}$, Irinel Popescu ${ }^{1}$
}

Corresponding author: Irinel Popescu, MD, FACS, FEBS Professor of Surgery "Dan Setlacec" Center of General Surgery and Liver Transplantation Fundeni Clinical Institute

258, Sos. Fundeni, 022328

Bucharest, Romania

E-mail: irinel.popescu@icfundeni.ro

Received: 10.05.2019

Accepted: 12.06 .2019

Copyright () Celsius Publishing House www.sgo-iasgo.com
${ }^{1}$ Center of General Surgery and Liver Transplantation, Fundeni Clinical Institute, Bucharest, Romania

${ }^{2}$ Department of Oncology, Fundeni Clinical Institute, Bucharest, Romania

${ }^{3}$ Department of Anesthesia and Intensive Care, Fundeni Clinical Institute, Bucharest, Romania

\section{ABSTRACT}

Anatomical central hepatectomy is technically demanding and is often excessive. It has an increased risk for insufficient remnant liver volume, especially in case of P8 dorsal pedicle for segment 7 and/or P5 dorsal for segment 6 , and/or abnormal background liver. On the contrary, limited central hepatectomy (LCH) for centrally located tumors, based on preserving the P8 dorsal and some of P5 and P4 pedicles (depending on tumor placement) is conservative, and therefore has a low risk for insufficient remnant liver volume. It is less technically demanding, when compared to anatomical central hepatectomy. The right side of the resection plane is driven along the P8 dorsal pedicle intersecting the P8 ventral pedicle and as few of the P5 pedicles as possible. The left side of the resection plane is established according to tumor placement anywhere in between the Cantlie's plane and the falciform ligament. The video presents 5 cases that support the conclusion that LCH may be standardized, with good results, especially when using intraoperative ultrasound guidance. Key words: liver resection, intraoperative ultrasound guidance, central hepatectomy

\section{Background}

Anatomical central hepatectomy $(\mathrm{S} 4+\mathrm{S} 5+\mathrm{S} 8)$ :

- Often excessive (as all major hepatectomies), according to the modern concept of conservative liver surgery;

- Risk for insufficient remnant liver volume, in case of: - P8d for S7 (approx. 45\%), or P8d for S7 and P5d for S6 (approx 20\%) ${ }^{1}$ - abnormal background liver (steatosis, cirrhosis);

- Technically demanding (hilar approach, RHV exposure); 


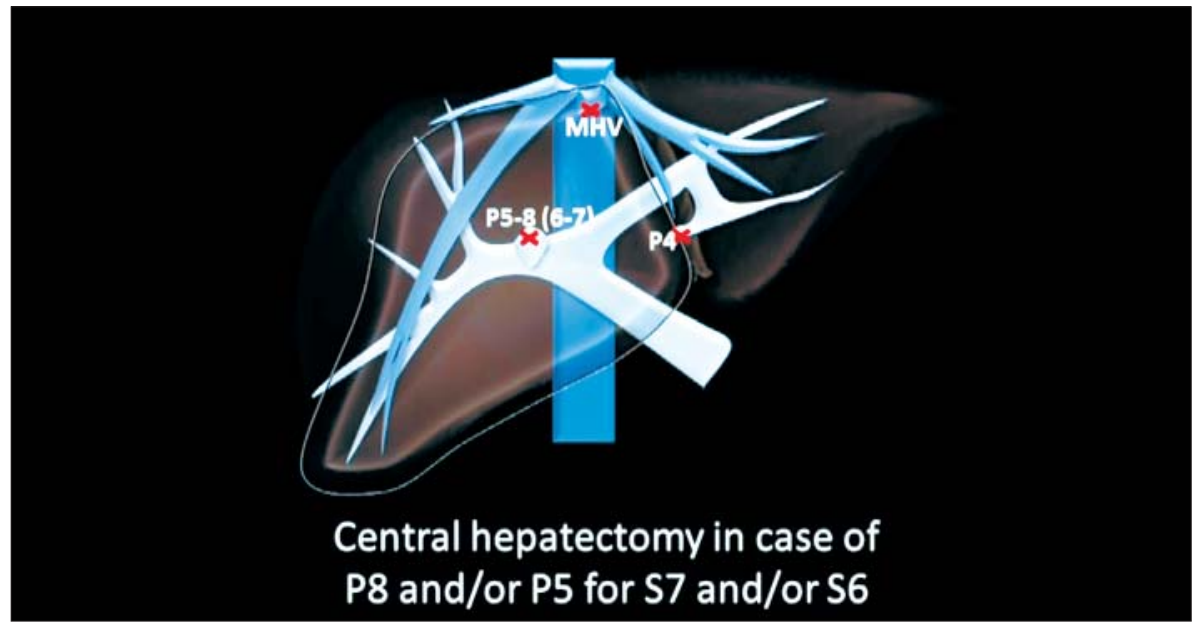

\section{Limited central hepatectomy ( $\mathrm{LCH})$}

- based on preserving P8d, some of P5 \& P4 pedicles depending on tumor(s) placement

- conservative - no sectional pedicle approach;

- no risk for insufficient remnant liver volume

- less technically demanding - no hilar approach, no RHV exposure;

- facilitated by intraoperative ultrasound (IOUS) guidance

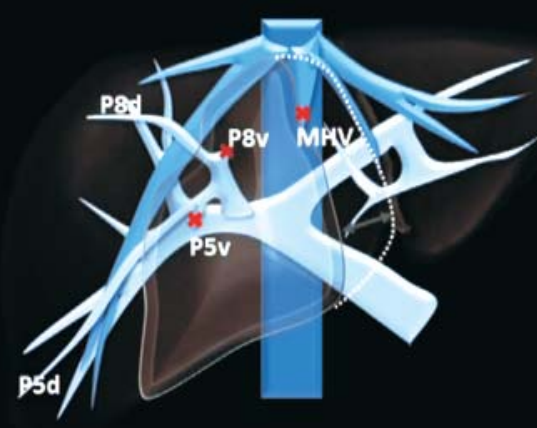

Limited central hepatectomy

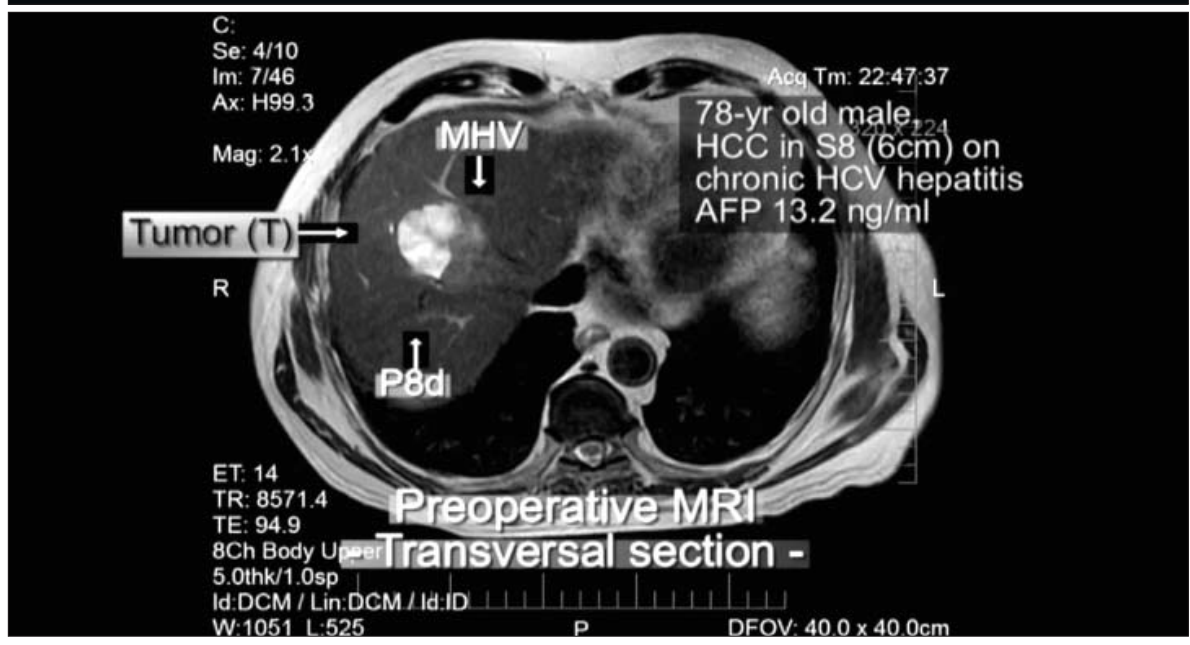



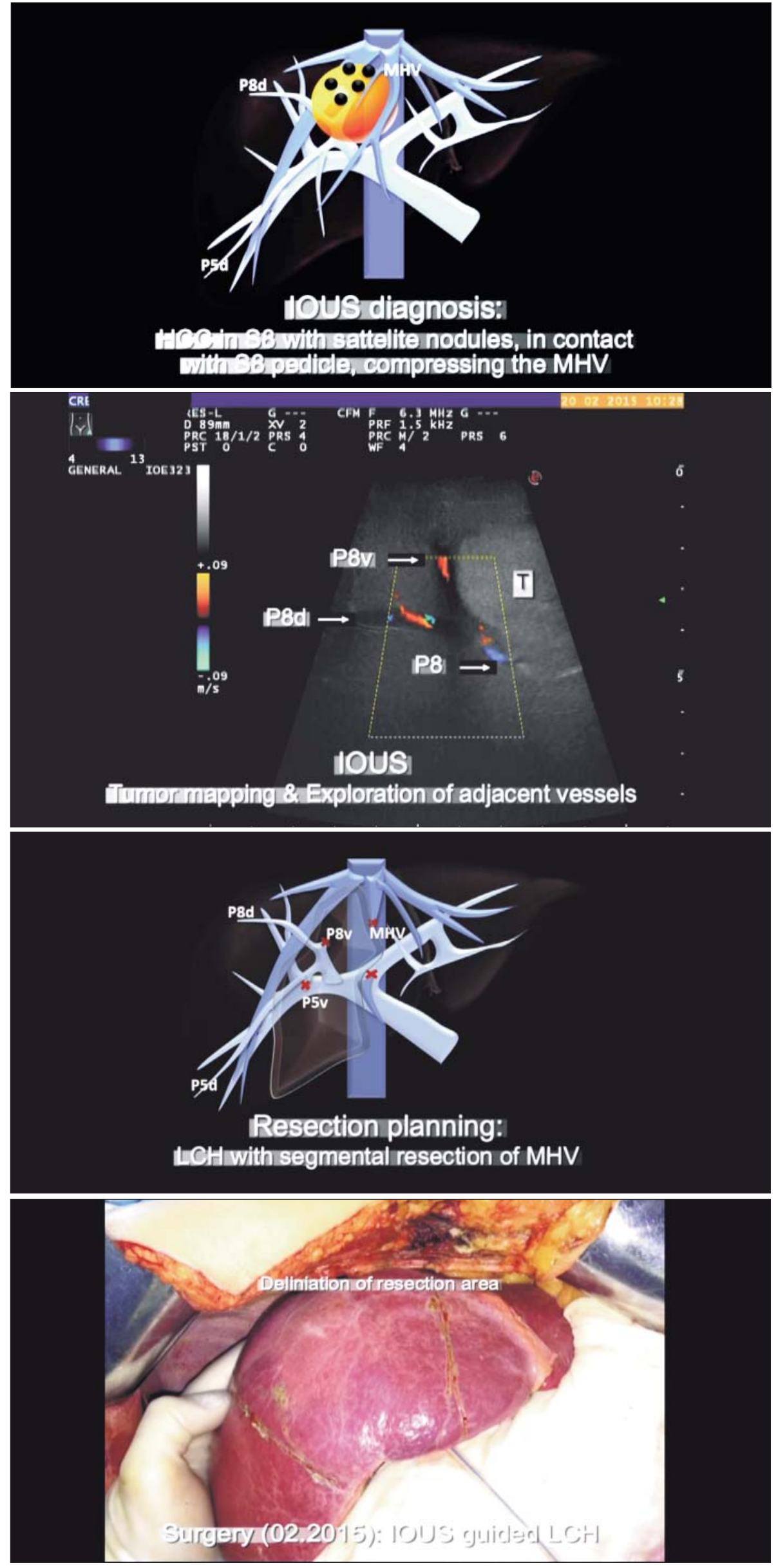

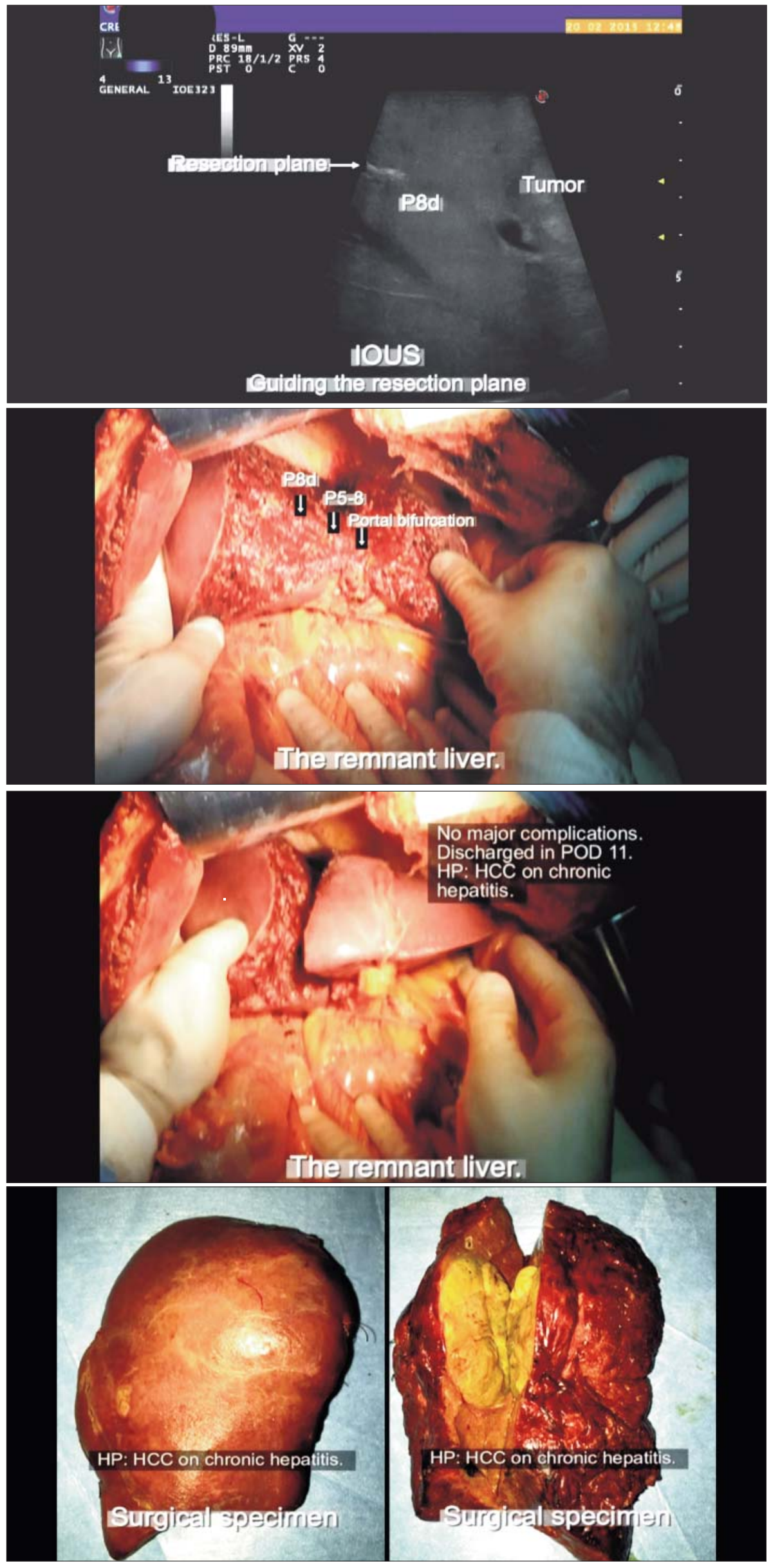

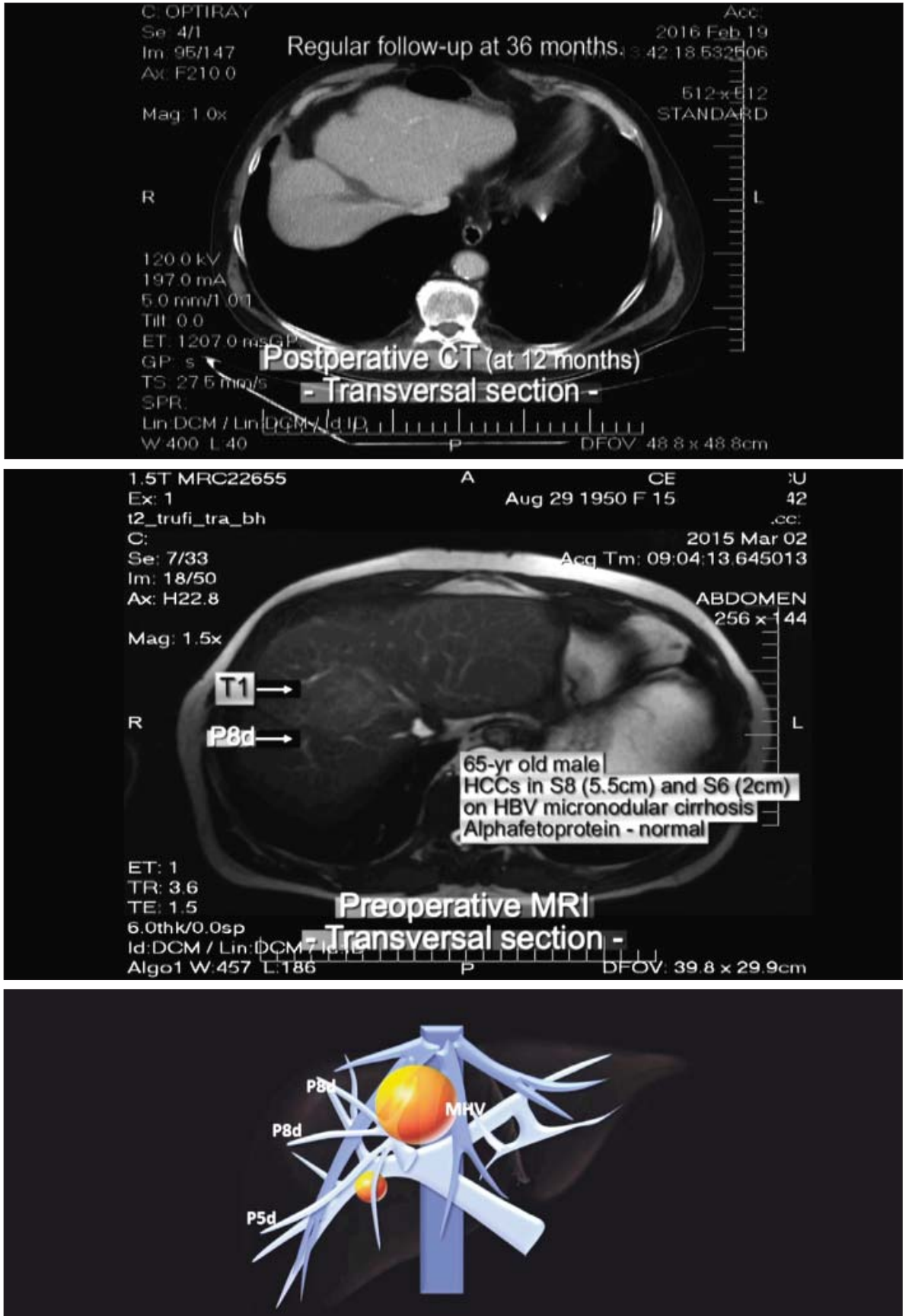

HCCs in $\mathbf{5 8}(5.5 \mathrm{~cm})$ in contact with P8d and P8 pedicles, and $\mathrm{S6}(2 \mathrm{~cm})$ in contact with $\mathrm{P} 6$ pedicle.

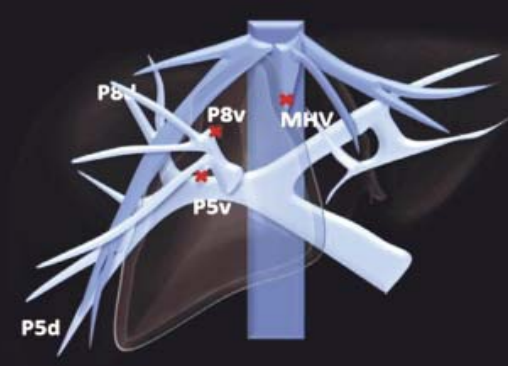

LCH extended to S6 and S1 

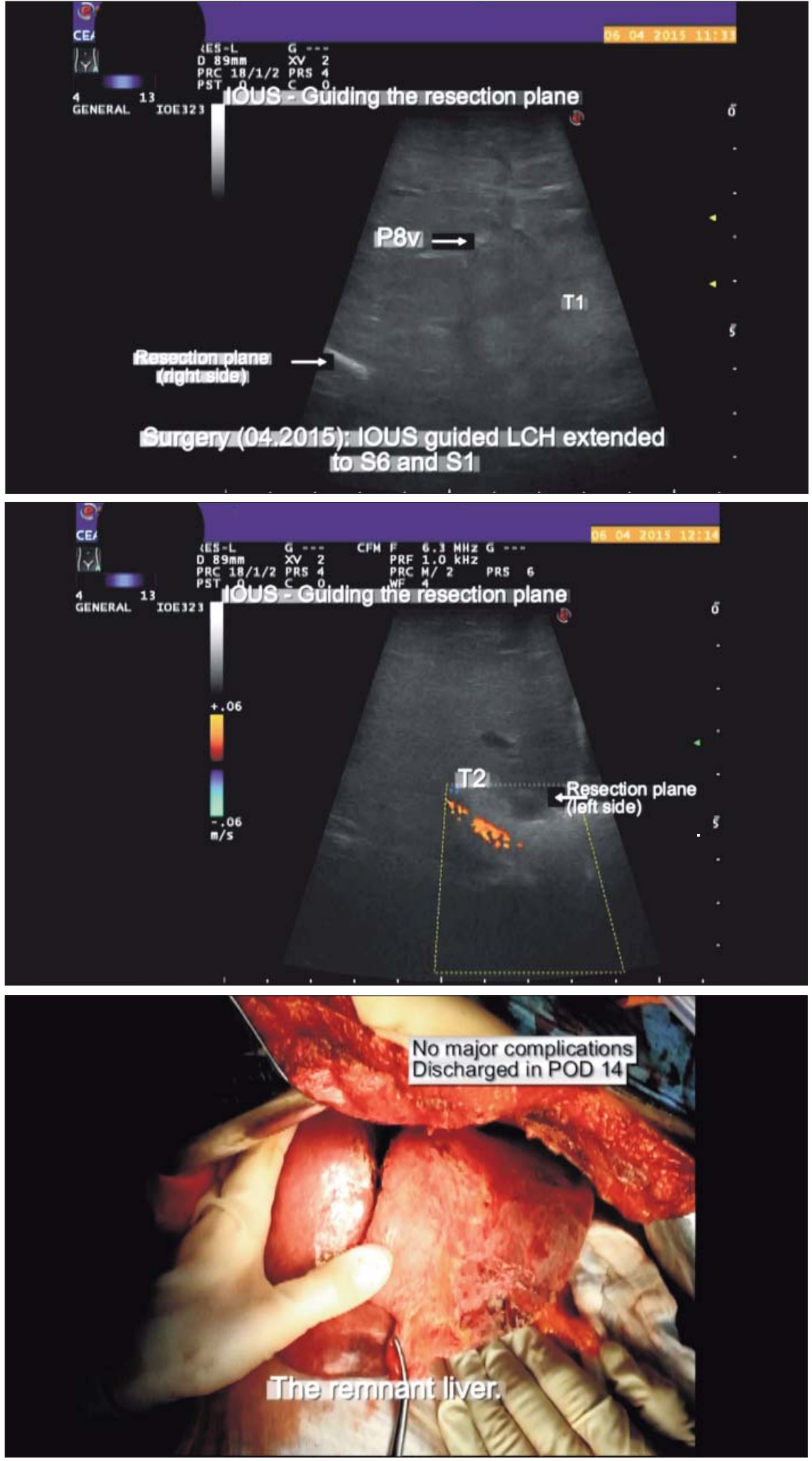

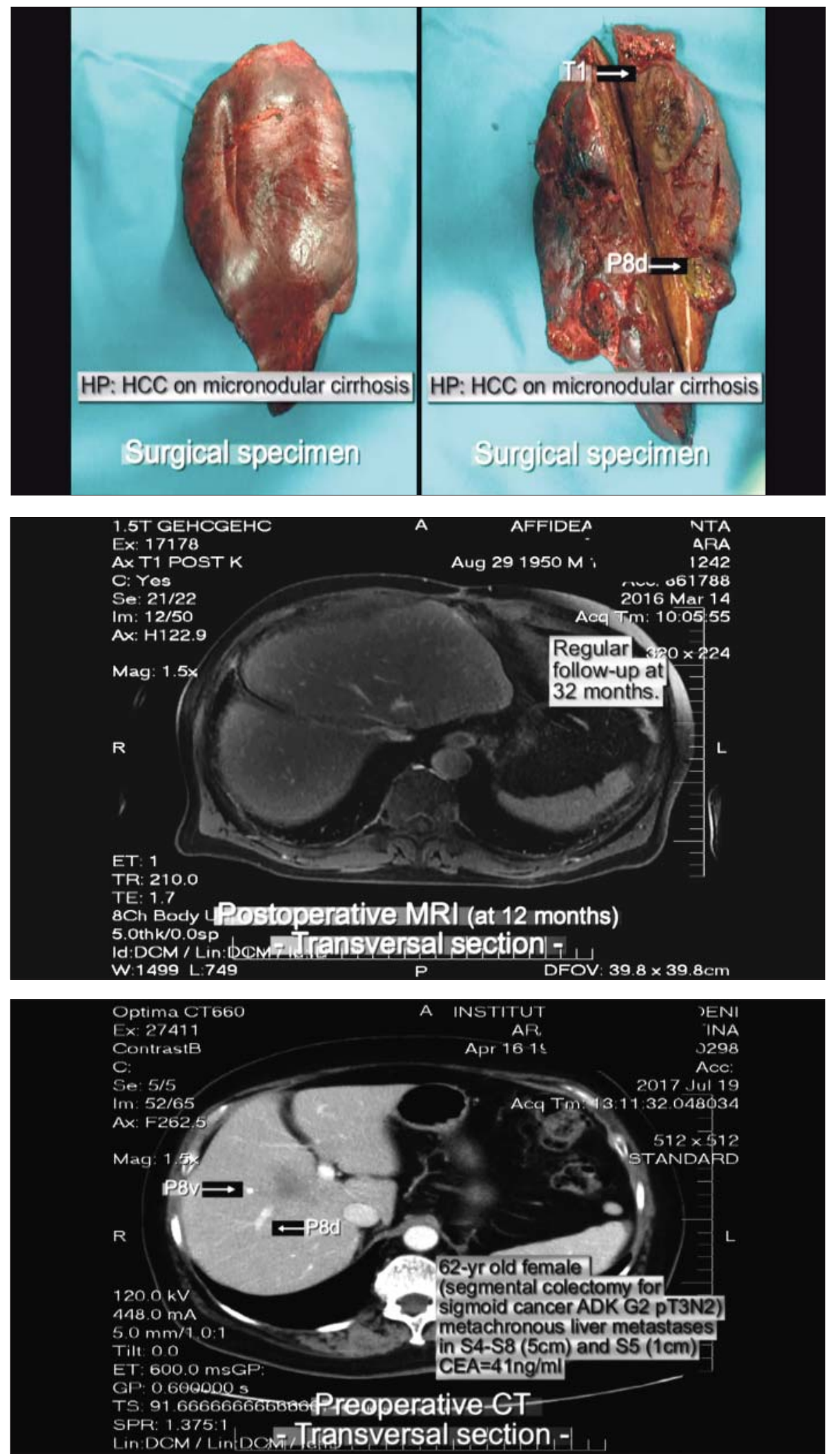

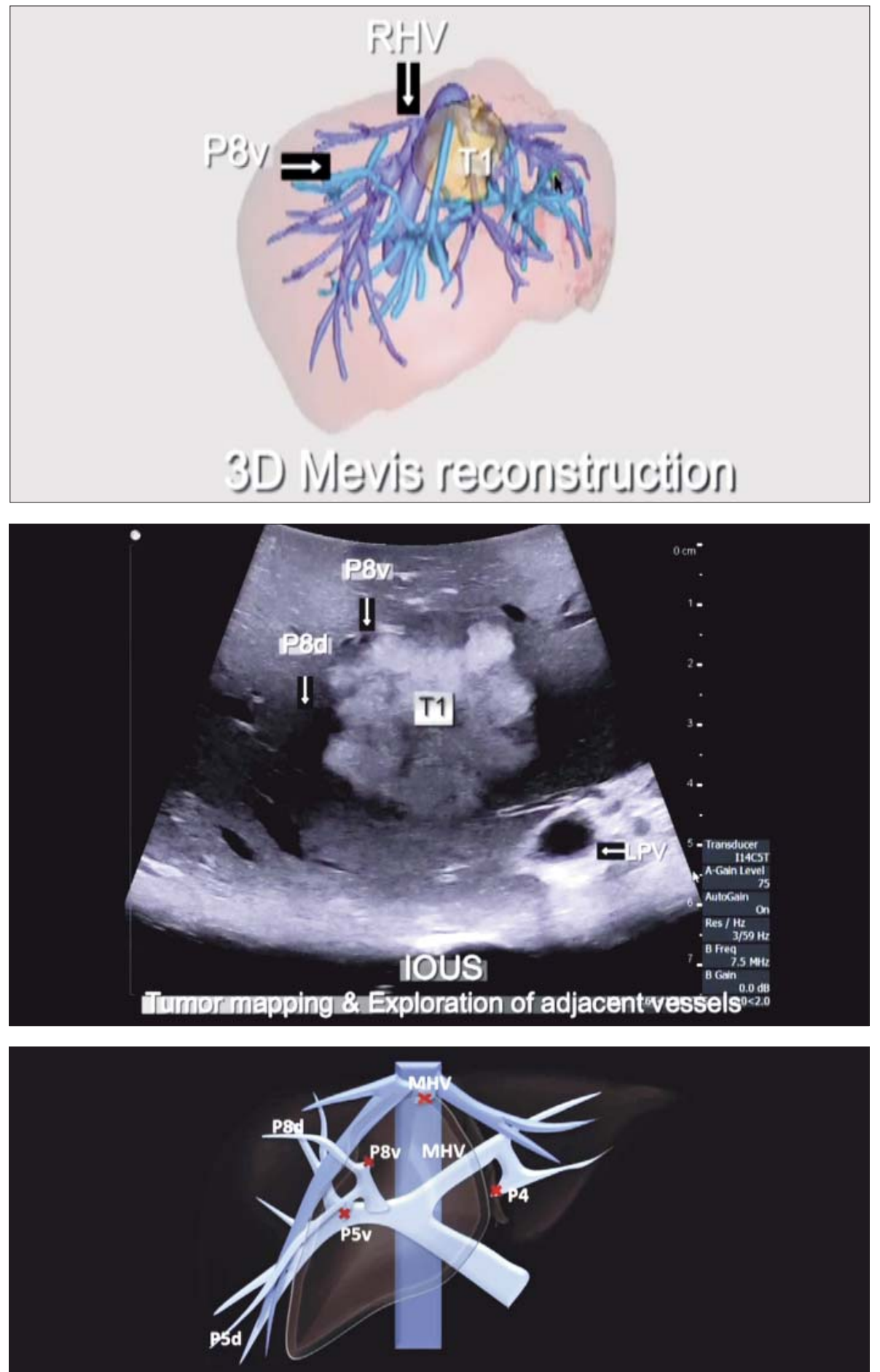

\section{LCH extended to S1}



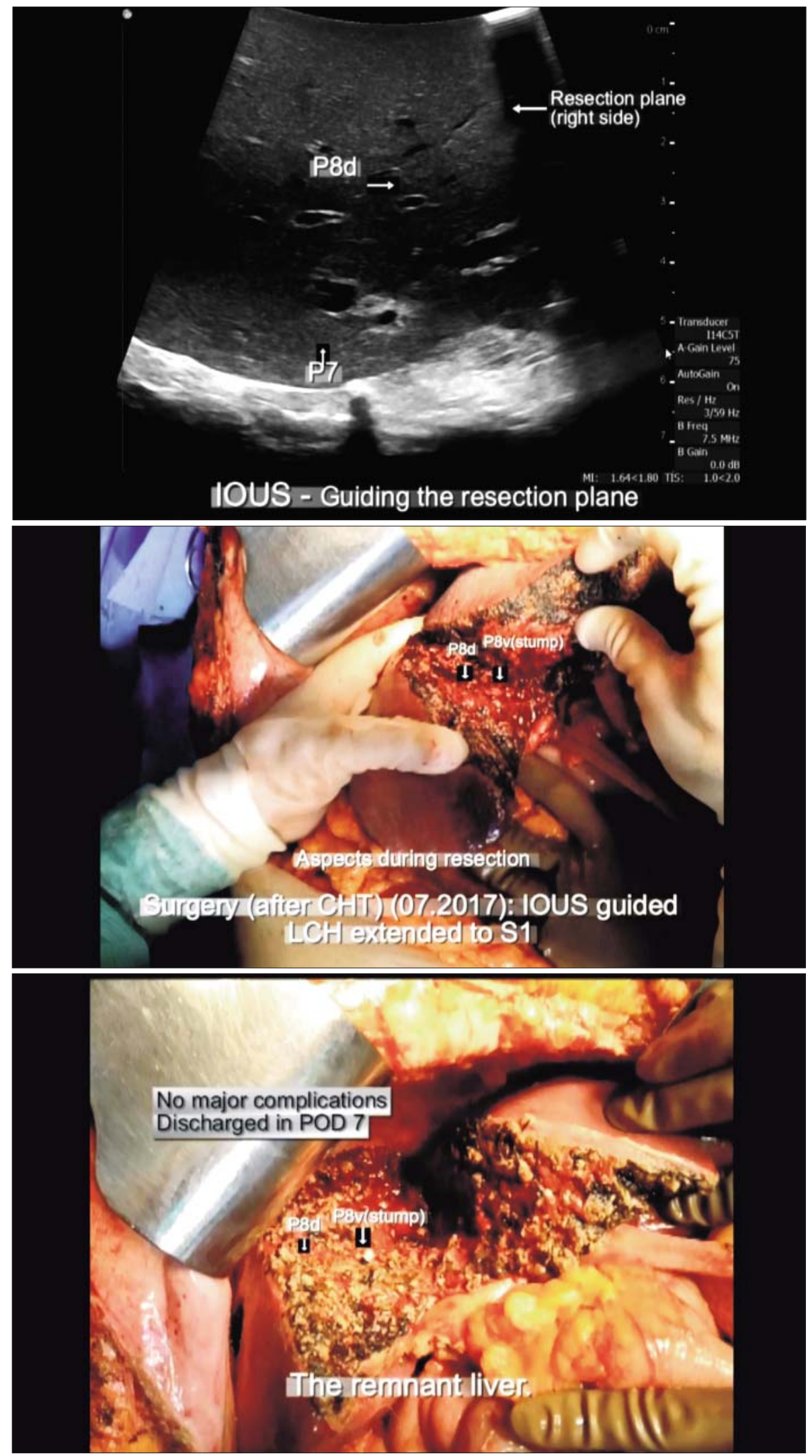

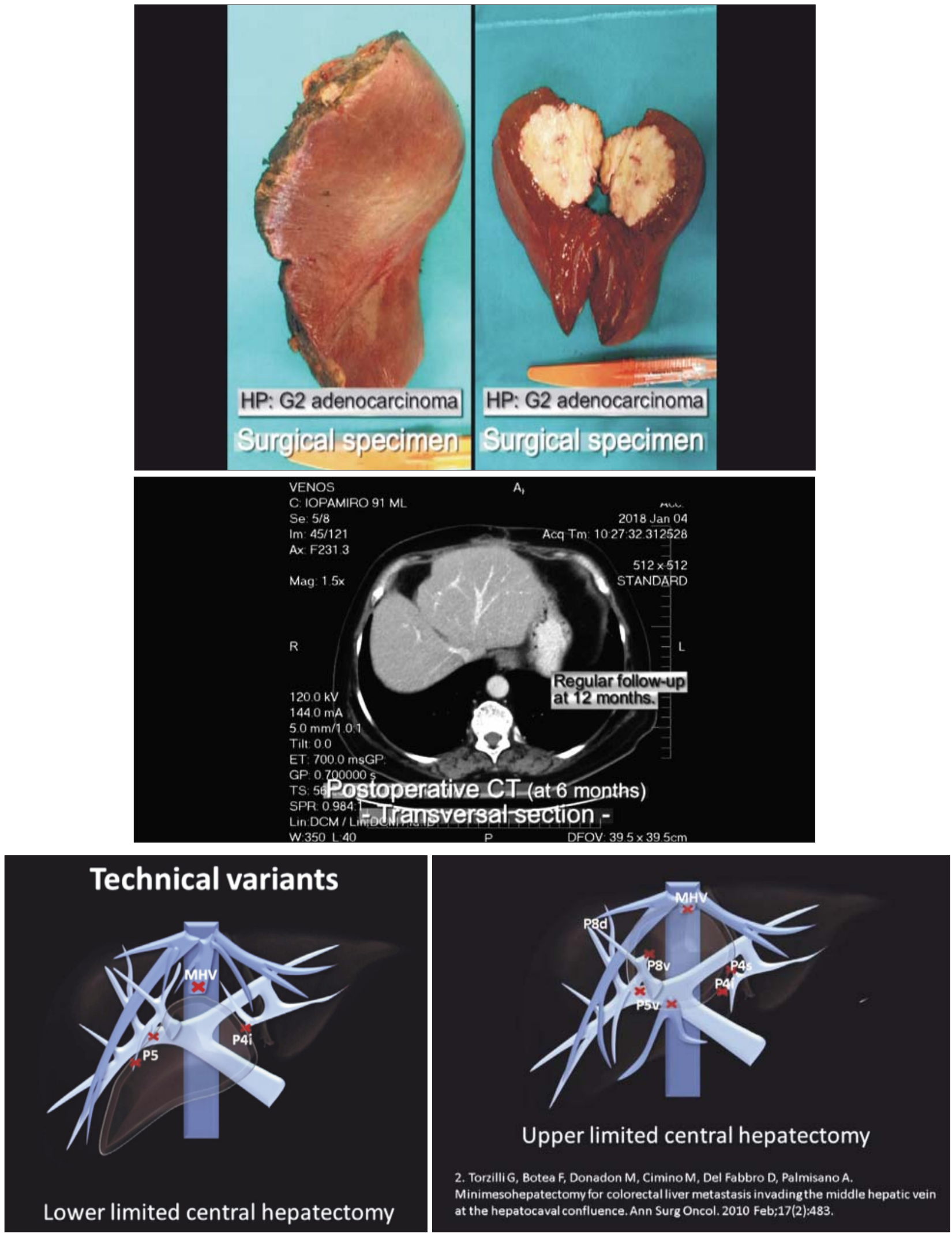

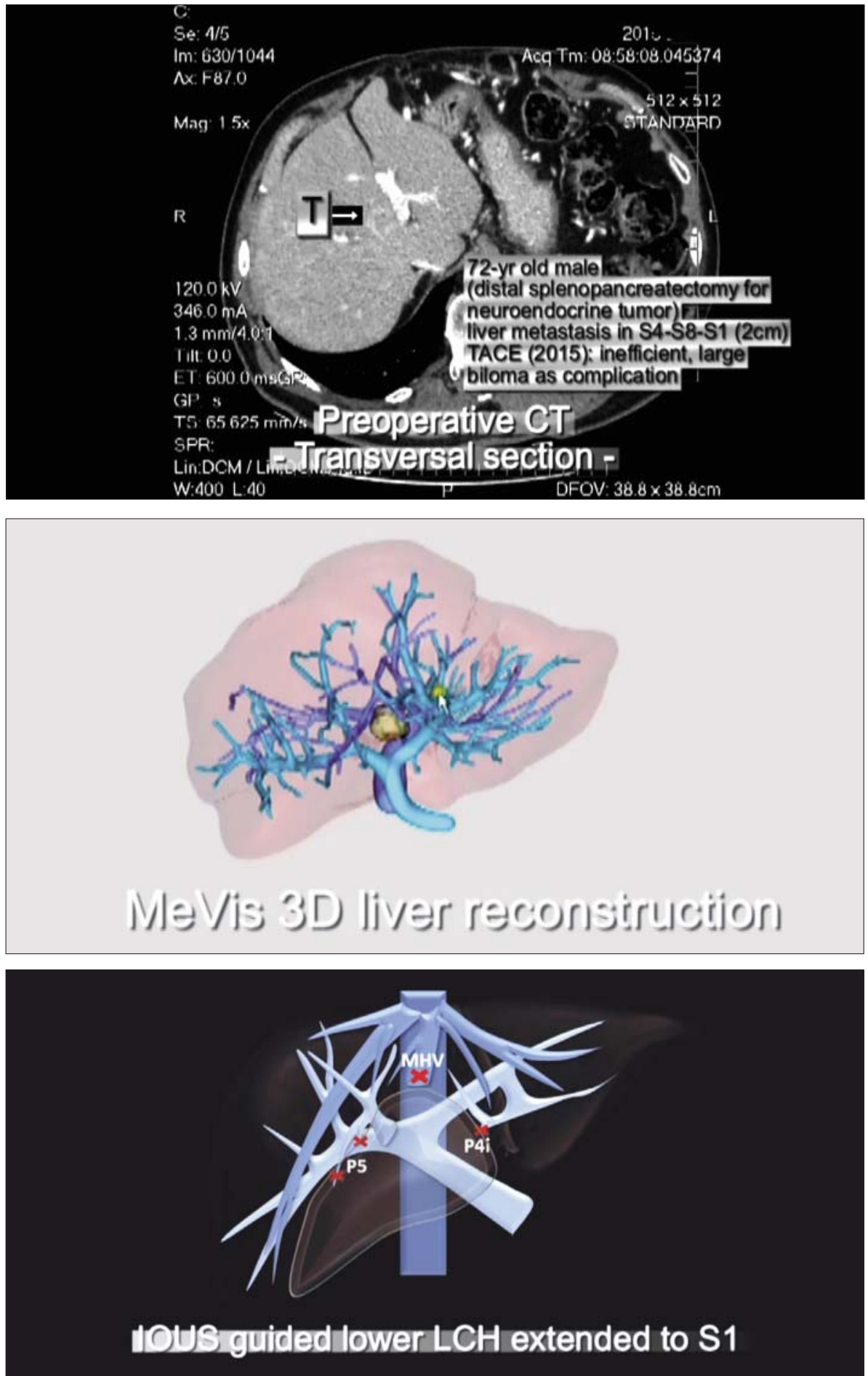

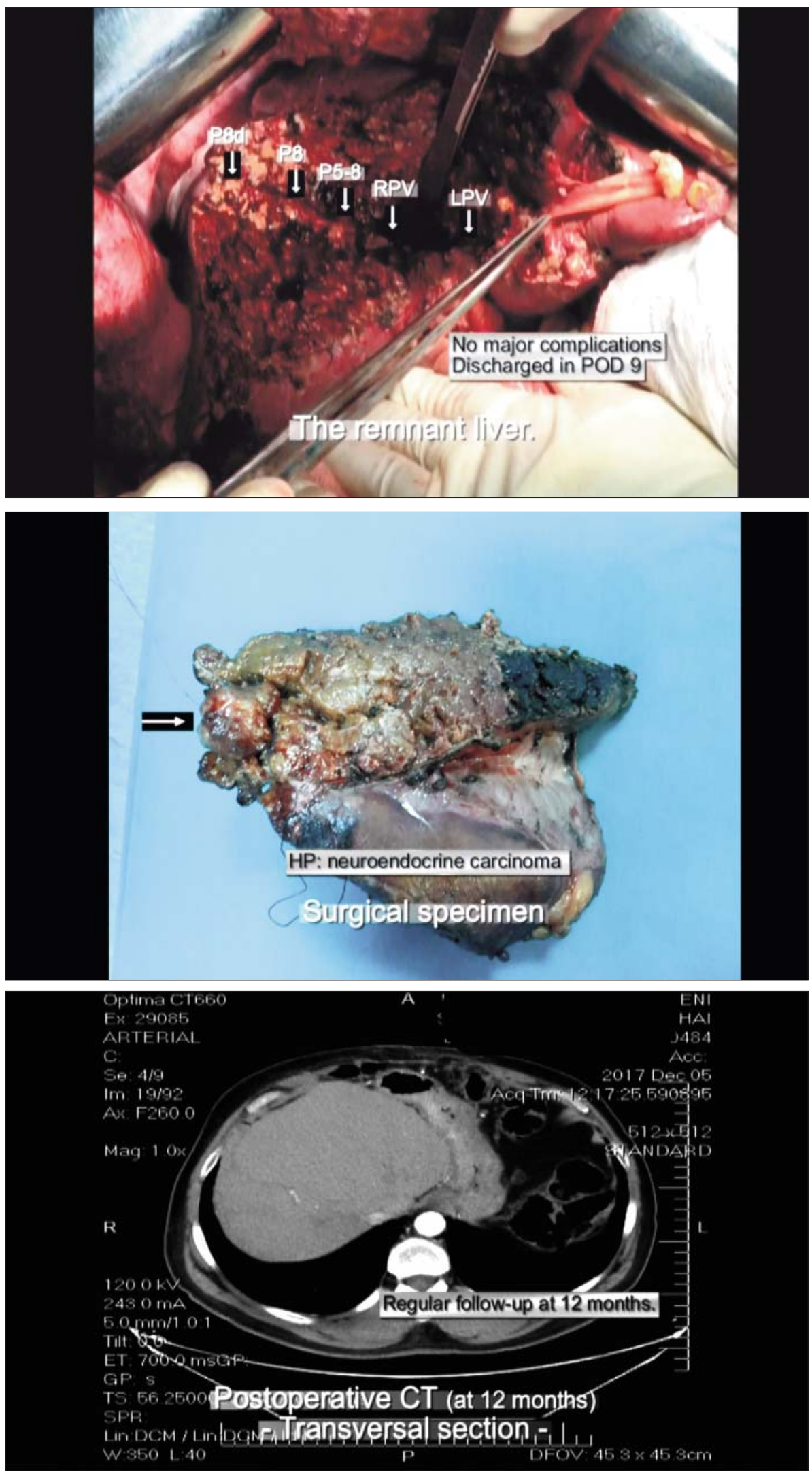

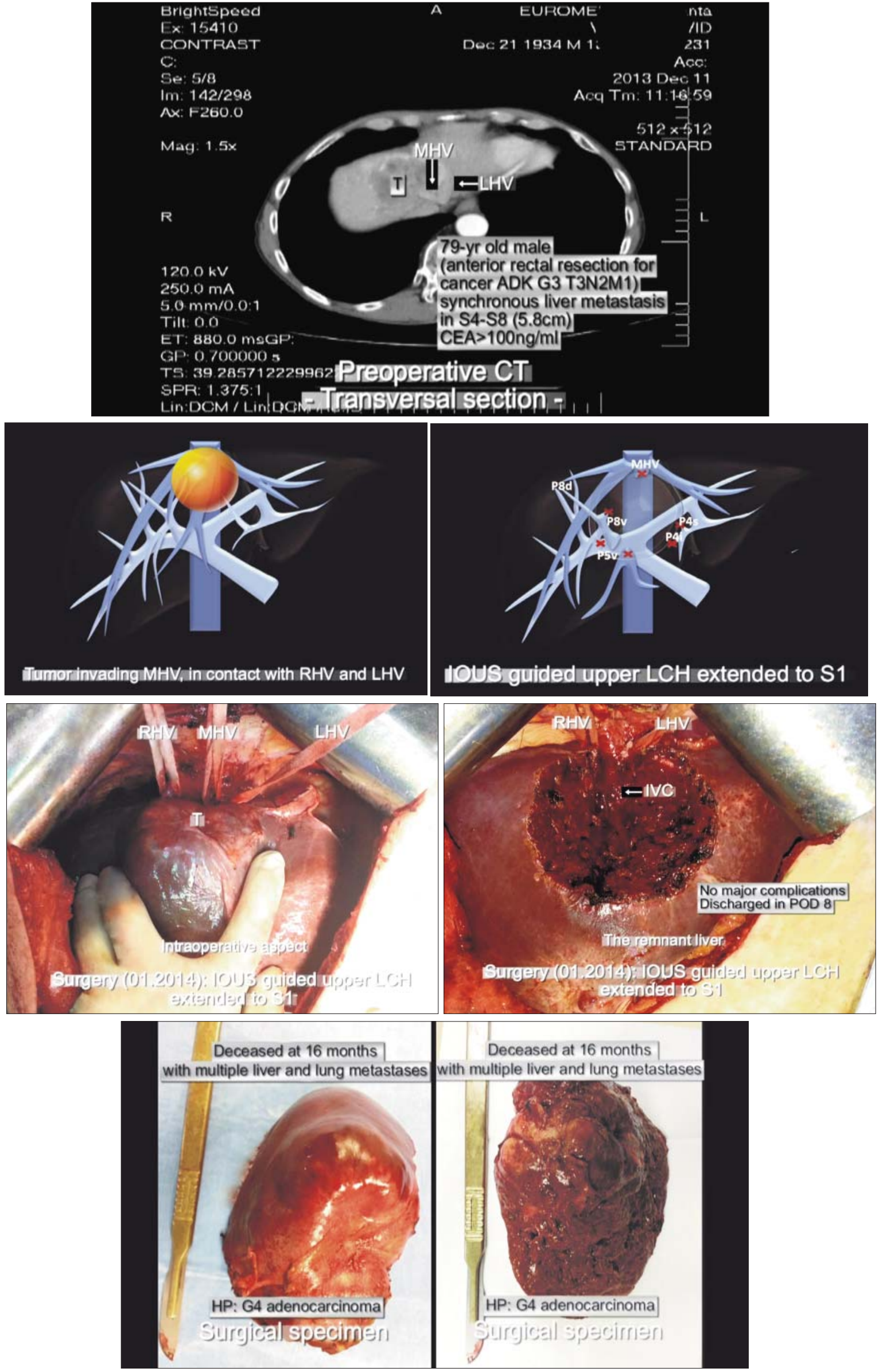


\section{Conclusions}

- Limited central hepatectomy:

- Feasible

- Low morbidity and mortality

- May be standardized, especially when using IOUS guidance for mapping and intersecting subsegmental pedicles

\section{Conflict of interest}

All author declare that they have no conflict of interest.

\section{REFERENCES}

1. Won TW, Park DE, Lee YH, Chae KM. A new classification of the right portal vein using 64 channel multi-dectector CT (MDCT). J Korean Surg Soc 2008;75:96-101.

2. Torzilli G, Botea F, Donadon M, Cimino M, Del Fabbro D, Palmisano A. Minimesohepatectomy for colorectal liver metastasis invading the middle hepatic vein at the hepatocaval confluence. Ann Surg Oncol. 2010;17(2):483. 